\title{
Editorial
}

\section{Testbeds for Future Wireless Networks}

\author{
Jorge Navarro-Ortiz $\mathbb{D},{ }^{1}$ Cristina Cervelló-Pastor $\left(\mathbb{D},{ }^{2}\right.$ Giovanni Stea $\mathbb{D},{ }^{3}$ \\ Xavier Costa-Perez, ${ }^{4}$ and Joan Triay ${ }^{5}$
}

${ }^{1}$ Universidad de Granada, Granada, Spain

${ }^{2}$ Universitat Politècnica de Catalunya, Barcelona, Spain

${ }^{3}$ Università di Pisa, Pisa, Italy

${ }^{4}$ NEC Laboratories Europe, Heidelberg, Germany

${ }^{5}$ DOCOMO Communications Lab. Europe, Munich, Germany

Correspondence should be addressed to Jorge Navarro-Ortiz; jorgenavarro@ugr.es

Received 2 May 2019; Accepted 5 May 2019; Published 16 June 2019

Copyright ( 2019 Jorge Navarro-Ortiz et al. This is an open access article distributed under the Creative Commons Attribution License, which permits unrestricted use, distribution, and reproduction in any medium, provided the original work is properly cited.

In the last years, the growth of wireless traffic has been enormous. For instance, according to the latest CISCO forecast, the number of mobile devices will grow from 8 billion in 2016 to 11.6 billion in 2021, including 3.3 billion M2M connections. Similarly, other wireless technologies such as Wi-Fi-with 541 million public hotspots by 2021-and LPWAN-accounting for 31\% of M2M devices by 2021-will also experience a massive increase.

The scientific literature dealing with present and future wireless networks is indeed abundant. A large part of it focuses on formal concepts and theoretical architectures, which are evaluated via analytical models or simulation. Although theoretical works are indispensable for the advancement of knowledge and innovation, testbeds and prototypes are essential to demonstrate the correct operation and performance of these technologies.

Designing and constructing testbeds and prototypes is a non-trivial endeavor, which often requires joint expertise from different engineering areas (such as signal processing, electronics, networking, and software). Sound measurement and test methodologies are required and must be documented. The practical insight gained in setting up a testbed - such as lessons learned, dos and do nots - is often very relevant to researchers and practitioners. Moreover, testbeds and prototypes are in fact enablers of further research, acting as catalyzers of inter-institution collaborations.

This special issue is to explore recent advances and stateof-the-art research related to the development of testbeds and prototypes for relevant wireless and mobile technologies, including radio, software, and architectural aspects. The response to our call for papers for this special issue was satisfactory, and we finally selected a total of six high-quality papers, after a thorough peer-review process.

Software-defined Networking (SDN) is nowadays one of the main network architecture paradigms used for building experimentation testbeds. Even more, SDN principles are taken as a foundation to re-engineer networks. One good example in the present special issue is the paper entitled "Design and experimental validation of a software-defined radio access network testbed with slicing support", by Aikaterini K. Koutlia et al. The paper describes the key design and implementation aspects of an experimental testbed of a Software-defined Radio Access Network (SD-RAN) with slicing support. In the testbed, an SD-RAN Controller aggregates part of the RAN control plane functionality (e.g., slice-aware/multi-cell admission control) together with RAN slicing management functionality. The functional design of the testbed is in line with 3GPP Release-15 specifications related to information modeling and network slicing management. The testbed is used to demonstrate the provisioning of RAN slices (i.e., preparation, commissioning, and activation phases) and the operation of the implemented Radio Resource Management (RRM) functionality for sliceaware admission control and scheduling with commerciallyavailable user equipment (UE). The clearer decoupling of control and forwarding planes in SDN, and the flexibility 
that such approach brings enabling centralized network control, are further explored by Ricardo Santos et al. in the paper entitled "MmWave backhaul testbed configurability using software-defined networking". In the paper, the authors propose a Software-defined Networking approach to enable a wireless backhaul for small cells. The wireless backhaul is formed by a mesh of millimeter-wave links with multihop paths, which can be dynamically reconfigured to interconnect the cells. This solution enables the provision of a multigigabit and low-latency interconnection without using optical links, which may not be economically feasible for large-scale deployments. The proposed network architecture, named SOCRA, makes the SDN control plane responsible for configuring not only the data plane forwarding, but also the link configuration, antenna alignment, and adaptive node power-on/off operations. The performance evaluation of the implemented testbed shows that an optimal channel assignment between the mesh links can result in a $44 \%$ throughput increase, when compared to a sub-optimal configuration. In the implementation of the testbed, diverse types of commercially available equipment (such as minicomputers and power units) and open source software components are employed. Finally, Hai Xue et al. propose two packet scheduling schemes, First Come First Serve-Pushout (FCFS-PO) and FCFS-PushOut-Priority (FCFS-PO-P) to effectively handle the overload issue of multiple-switch SDNs targeting the edge-computing environment in the paper entitled "Packet scheduling for multiple-switch software-defined network in edge-computing environment". After developing analytical models for their operation, extensive experiments on a real testbed are carried out. The proposed schedulers outperform the classical FCFS-Block (FCFS-BL) in terms of packet waiting time. Furthermore, FCFS-PO-P achieves better performance in terms of sojourn and waiting time for various traffic conditions.

This special issue also includes interesting articles that employ the Network Functions Virtualization (NFV) paradigm to virtualize and orchestrate the required network services. Similar to SDN, NFV is one of the enablers for future wireless networks such as $5 \mathrm{G}$. In the context of the Internet of Things (IoT), Jorge Navarro-Ortiz et al. present in the paper "A LoRaWAN testbed design for supporting critical situations: prototype and evaluation" a selfhealing LoRaWAN network architecture to provide resilience when part of the equipment in the core network becomes faulty. Resilience is achieved by virtualizing and properly orchestrating the different network entities. Different options have been designed and implemented as real prototypes. Based on the performance evaluation, the authors claim that microservice orchestration along with several replicas allows the network administrators to seamlessly recover after a catastrophic situation. Focusing on Unmanned Aerial Vehicles (UAVs), Christian Tipantuña et al. introduce an optimal drone scheduling algorithm in paper "An NFVBased Energy Scheduling Algorithm for a 5G Enabled Fleet of Programmable Unmanned Aerial Vehicles", which, by leveraging 5G and NFV capabilities, is able to perform an efficient energy-aware management of resources for network services provisioning. Simulation results validate the proposal and evaluate its performance in terms of both results (i.e., achieved metrics), and costs (i.e., the amount of resources needed for the execution of services in different scenarios). A testbed with several Raspberry Pi 3B and a specific power meter has been employed to measure power consumption under the simulated conditions. Future work includes the modelling of the services availability and the design of heuristic approaches.

Finally, one of the works highlights the importance of these testbeds and prototypes in a European funded project. In particular, in the paper "QoE evaluation: the TRIANGLE testbed approach", Almudena Diaz Zayas et al. describe a testbed, the test methodology, and the set of test cases developed within the TRIANGLE project, with the objective of testing and benchmarking mobile applications, services, and devices. The testbed can be remotely accessed through a web portal or through a Keysight Testing Automation Platform for advanced users. A set of test cases has been implemented that specify the measurements that should be collected to compute the Key Performance Indicators of the feature under test, which are then normalized into a standard 1-to-5 scale, the one typically used in Mean Opinion Scores (MOS). These synthetic MOS values are finally aggregated to obtain a synthetic MOS score in each test case. The paper includes a summary of these scores for the considered scenarios.

\section{Conflicts of Interest}

The editors declare that they have no conflicts of interest regarding the publication of this special issue.

\section{Acknowledgments}

The editors thank all of the authors for their submissions to this special issue. We hope that this special issue will further encourage research interests in the area of wireless network testbeds and prototypes.

\author{
Jorge Navarro-Ortiz \\ Cristina Cervelló-Pastor \\ Giovanni Stea \\ Xavier Costa-Perez \\ Joan Triay
}




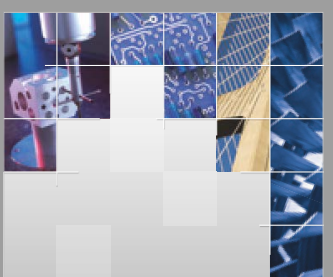

\section{Enfincering}
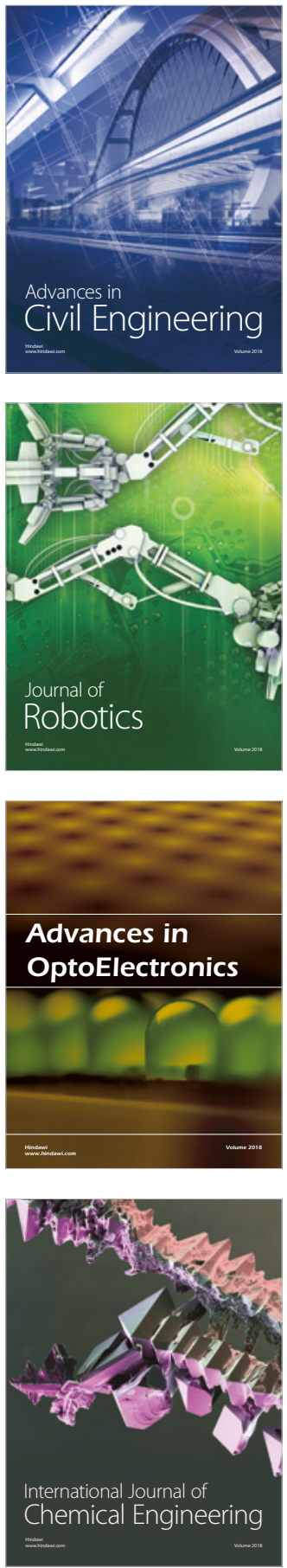

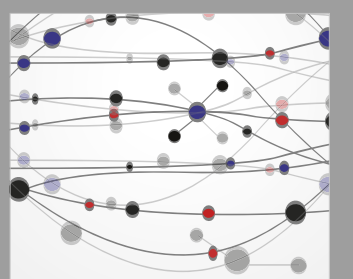

\section{Rotating \\ Machinery}

The Scientific World Journal

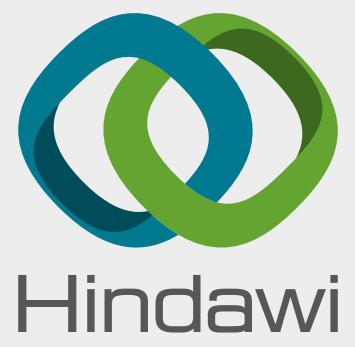

Submit your manuscripts at

www.hindawi.com
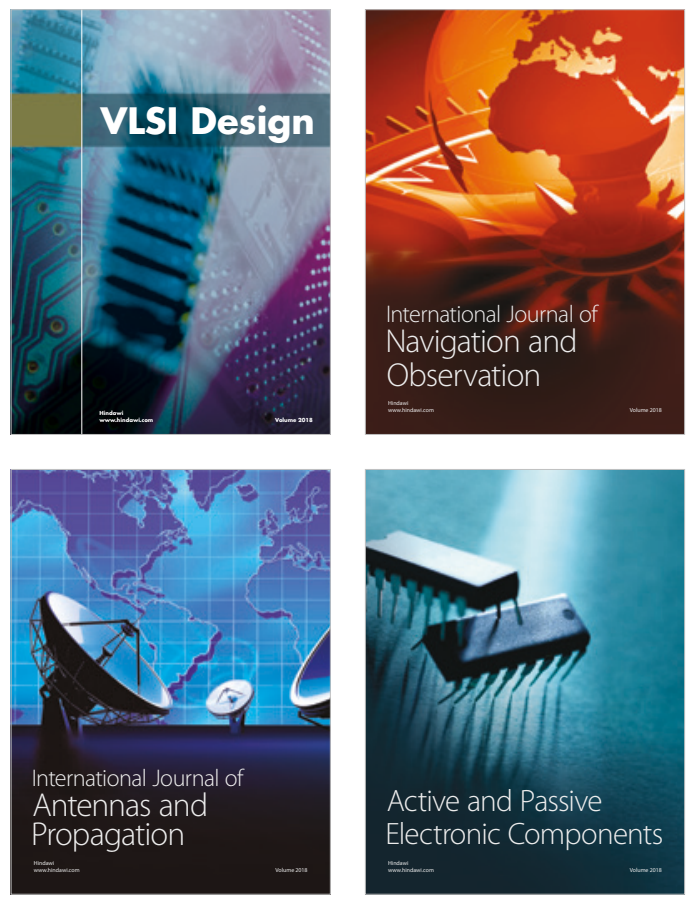
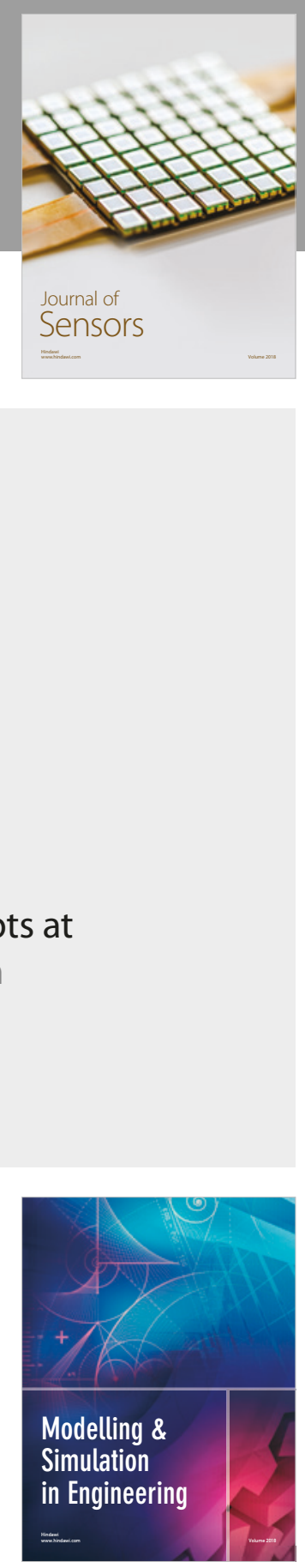

\section{Advances \\ Multimedia}
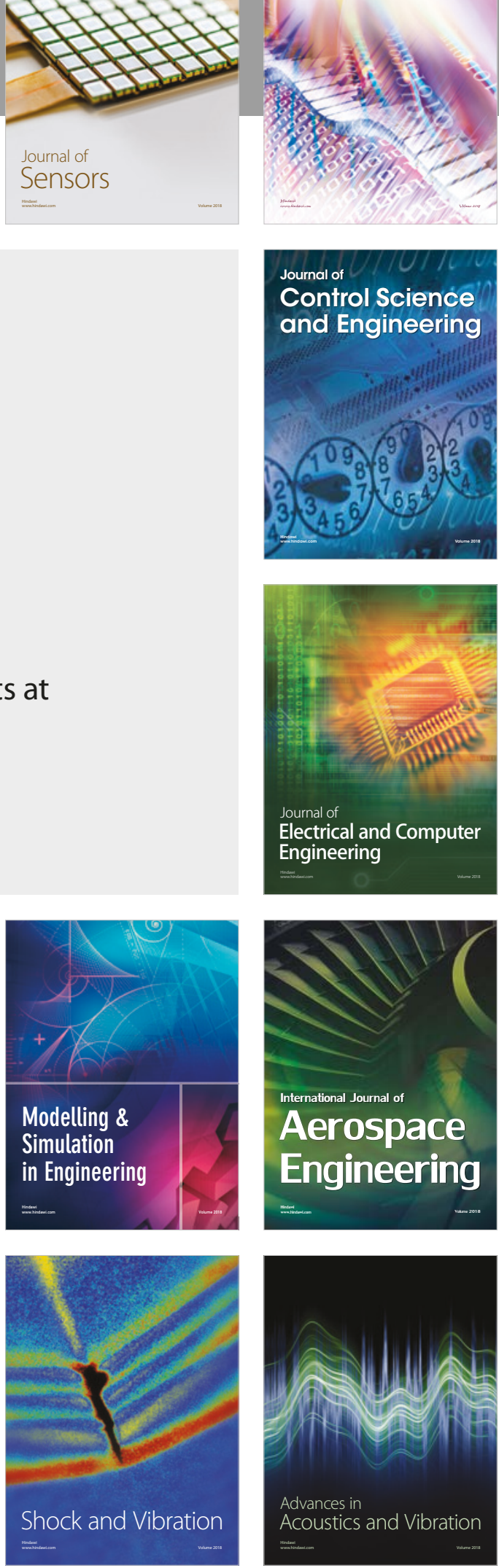